\title{
Der freundliche Halbautomat
}

\section{Andreas Gutzeit}

Facharzt Radiologie, MaHM, Zürich

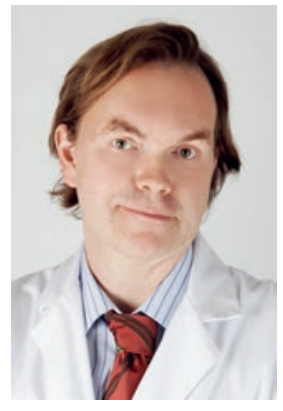

Ruhe nach dem Sturm. Vier Tage Nachtdienst im Spital vorbei. Ich sitze erschöpft im Zug und fahre nach Hause, Montagmorgen, 9.01 Uhr im Zürich HB auf Gleis 6. Von draussen dringt das Quietschen von Transportwagen, angefüllt mit Getränkekisten, Sandwiches und Stapeln WC-Papier. Die aufgehende Sonne scheint durch die Glasscheibe auf meine Haut, der Nachtdienst ist nicht spurlos an mir vorbeigegangen. Im Spiegelbild erahne ich meine dunklen Augenränder, meine Bartstoppeln. Auf dem Bahnsteig eilen Fahrgäste an mir vorbei, um den Zug zu erreichen.

Mir gegenüber sitzt ein jüngerer Mann: sommerliches Outfit, wie mit dem Lineal gezogener Haarschnitt. In der einen Hand hält er eine Tasse Kaffee, mit der anderen ein Buch mit dem Titel: «Künstliche Intelligenz: China, Silicon Valley, und die neue Weltordnung» von Kai-Fu Lee. In den letzten Jahren hörte und las ich von IT- und Google-Experten und Repräsentanten des Silicon Valley, dass Ärzte, die das Potential der neuen digitalen Techniken und Algorithmen nicht bedingungslos umsetzten, zukünftig keine Chance auf dem Gesundheitsmarkt haben werden: also Ärzte wie ich. Nach deren Prognose werde ich als Radiologe in den nächsten Jahren meine Arbeit verlieren, da die neuen Techniken auf Knopfdruck Diagnosen ausspucken, selbstverständlich meist besser als meine. Zumindest sagt das die Werbung, aber warum sehe ich keine solchen Geräte in der Praxis? Vor fünf Jahren sagte man uns, dass heute kein Auto mehr ohne autonome Fahrweise fahren würde - die Realität ist anders. Diese neuen IT-Propheten suchen uns Ärzte auf Kongressen auf, wollen uns über Supercomputer, OP-Roboter, Künstliche Intelligenz, 3D-Brillen aufklären und schleudern uns Slogans zu, als wären es bunte Bälle für Kinder: «Nehmt die Künstliche Intelligenz an, habt keine Angst vor ihr.»

Vor neuen Technologien habe ich keine Angst. Solange ich meinen Computer bei Vorträgen noch nicht problemlos an verschiedene Beamer anschliessen kann, weil die Stecker nie passen, oder Videos bei Kongressen oft nicht funktionieren, mache ich mir keine Sorgen, dass ein Computer komplexe ärztlichen Tätigkeiten in nächster Zeit übernehmen kann. In meinem Berufsleben sind Künstliche Intelligenz und Algorithmen weit weg, wirken aus Entfernung surreal. Würden unsere Patientinnen und Patienten Maschinen als Menschersatz tolerieren? Die Radiologie ist stets Vorreiter in der Medizintechnologie gewesen, aber trotz Technik und Maschinen werden menschliche Fachkräfte meiner Art zunehmend benötigt. Der Duft des Kaffees meines Sitznachbarn steigt mir in die Nase, ich fühle mich geborgen. Während ich meinen
Nachbarn anschaue, frage ich mich, wie die Medizin in 20 Jahren aussehen wird. Werden junge Menschen wie er unseren Alltag digital organisieren? Was wird meine Rolle als Mensch und Arzt sein? Braucht es mich mit meinen kritischen Fragen noch? Wird der Wert der menschlichen Zuwendung in digitalen Prozessen noch Platz finden? In einer meiner Studien konnte ich kürzlich zeigen, dass Patienten nach der Mammographie mit einem Radiologen die Befunde persönlich besprechen wollen. Sie wollen ein Gesicht, dem Patientinnen Enttäuschung und Hoffnung entgegenbringen können. Werden wir das als zukünftige alte Patienten nicht auch wollen? Ein Gesicht, menschliche Kommunikation und Zuwendung? Würde ich in ein Flugzeug steigen, wenn der Pilot nicht mehr im Cockpit wäre? Nach den beiden Abstürzen der Boeing737-MAX-Flugzeuge durch verrücktspielende Autopiloten habe ich Zweifel.

Das Knistern aus der Sprechanlage unterbricht meine Gedanken: «Sehr verehrte Fahrgäste, leider verzögert sich unsere Abfahrt. Grund dafür ist ein medizinischer Notfall. Falls sich im Zug ein Arzt oder andere medizinische Fachkräfte befinden, bitten wir sie umgehend, sich in Wagen 9 beim Zugpersonal zu melden.» Ich schaue mich einen Moment um, die Hoffnung, dass sich jemand erhebt und zu Wagen 9 eilt, ist vergeblich, und der junge Mann schaut mich fragend an. Mein Gewissen regt sich, aber ich zögere noch. Als Radiologe fühle ich mich für solche Fälle so kompetent, als würde ich gefragt, ob ein marodierender Problembär im Engadin geschossen werden soll oder nicht. Ich fühle mich kaum geeignet, aber gerufen. Ich gehe an den Abteilen des wartenden Zuges entlang. In Wagen 9 steht die Tür offen, und die Stimme der Kondukteurin bekommt ein reales Gesicht. Ihr rotes Halstuch mit dem SBB-Logo hängt schief, das Haar durcheinander, auf der Stirn Schweissperlen. "Sind Sie Arzt?», fragt sie mich. "Ich bin Radiologe.» Ihr fragender Blick erhellt sich, als ich nachschiebe: "Ja, ich bin Arzt.» Mit dem Arm in das Zuginnere gerichtet weist sie den Weg. Am Boden des Abteils liegt eine junge Frau in Seitenlage. Ich kann nur ihre Füsse sehen, da der Rest des Körpers unter eine Doppelsitzreihe gerollt ist. Neben ihr kniet ein junger Mann, er habe gestern den Ersthelferkurs für den Führerschein gemacht und berichtet, dass die Patientin plötzlich gekrampft habe und dann vom Sessel gefallen sei. «Der hat die gleiche Frisur wie der Mann mit dem Buch über Künstliche Intelligenz. Die jungen Leute sehen heute alle gleich aus», schwirrt es durch meinen Kopf. Das Betrachten der bewusstlosen Frau am Boden beendet den Gedanken. Sie liegt am Boden, der blaue 
Rock und die helle Bluse verrutscht, Beine und Füsse verdreht. Ihr Gesicht ist mir abgewandt. Ich erkenne, dass die Lippen blau schimmern. Ihre Halskette hängt schräg über dem Gesicht. Die Prüfung der Vitalparameter zeigt: Das ist kein Krampf, es ist ein Herzkreislaufstillstand. Mit Hilfe des jungen Mannes ziehe ich die Frau unter dem Sitz hervor, lege sie auf den Rücken und fange sofort mit der Herzdruckmassage an. Ich erteile knappe Anweisungen, lasse den Rettungsdienst anrufen und fordere den Defibrillator (AED) aus dem Bahnhof. Eine seltsame Ruhe überkommt mich. Der Druck auf den Brustkorb der Frau gleicht Wellen, die, regelmässig wie die Brandung am Meer, auf den Boden des Zuges prallen. Ihr linkes Auge ist halb geöffnet, es entwickeln sich eine Verbundenheit und ein Gefühl der Sorge um diese Frau, die ich vorher nie gesehen habe. «Du bist noch viel zu jung. Bitte stirb nicht!»

Pfeifen aus dem Mund entweicht durch Kompressionen des Brustkorbes. Das Abteil voller Menschen, die meisten lesen Gratiszeitung, wischen und tippen auf den Smartphones. Ich empfinde einen Widerspruch: Hier kämpft eine junge Frau, vielleicht eine Mutter, um ihr Leben, während die anderen Mitmenschen sich mit Banalem abgeben. Das Knacken der brechenden Rippen lässt mich erschaudern. Der AED erreicht uns, ich klebe die Kontakte auf den Brustkorb. Eine Stimme erklingt aus dem blinkenden AED. Ich überhöre die ersten Menüpunkte, bis ich das EKG sehen kann - eindeutig Kammerflimmern. Das Gerät bereitet eine erste Schockabgabe vor. Während das Gerät weiterredet, mache ich konzentriert weiter, drücke schweigend im Rhythmus, wie ich es oft trainiert habe. Die Stimme aus dem AED erscheint erneut: «Fester drücken.» Mitten im Geschehen spüre ich ein Tippen auf meiner Schulter, erkenne im Augenwinkel Metall auf meiner Schulter. War das ein Gänsekopf? Ich drehe mich um, hinter mir sitzt eine alte Dame, eleganter Zweireiher mit schwarzem Hut, der so gross ist wie das Sonnensegel der internationalen Raumstation ISS. Die Dame trägt einen Stock und tatsächlich, mit einem Gänsekopf als Knauf. Ich schätze sie auf 90 Jahre. «Entschuldigung, Herr Doktor. Wenn Sie ihn nicht aussprechen lassen, kann er Ihnen nicht helfen. Haben Sie verstanden? Sie sollen fester drücken»

Es dauert einen Moment, bis ich realisiere, was sie gesagt hat. Der grüne AED, der dauernd redet, spricht persönlich mit uns bzw. der alten Dame. Ich erkläre ihr, dass alles in Ordnung sei und dass ich Bescheid wisse. Aus einem Arztkoffer einer angrenzenden Praxis erhalte ich einen EpiPen, den ich durch die Strumpfhose in den Oberschenkel injiziere. «Zurücktreten, Patient nicht berühren, Schock abgeben", sagt das Gerät. Elektrisches Zischen erfüllt den Raum. Wir reanimieren weiter, obwohl die Patientin wieder eigene Atembewegungen aufweist. Das Kammerflimmern hat sich normalisiert, eine STStrecken-Hebung im EKG ist zu erkennen. Nach der Übergabe der Patientin an den Rettungsdienst danke ich dem jungen Helfer und schaue die ältere Dame an, vielleicht sogar leicht gekränkt, sicher aber irritiert und gleichzeitig immens erleichtert über die erfolgreiche Reanimation. Die Frau mit dem Gänsekopfstock scheint nicht ganz zufrieden zu sein.

«Gut, dass wir das Gerät hier hatten und es der Patientin geholfen hat, oder Herr Doktor?»

Sie, die schon fast ein ganzes Jahrhundert erlebt hat, scheint spontan eine persönliche Beziehung zum AED als Halbautomaten entwickelt zu haben. Habe ich selbst zu wenig gesprochen, zu wenige Emotionen gezeigt? Ist nicht nur unsere jüngste, sondern - entgegen allen Annahmen von Soziologen und Medienwissenschaftlern auch die älteste Generation unserer Gesellschaft bereits digital natives?

Zurück an meinem angestammten Platz, schaut der junge Mann kurz auf, nickt mir zu und vertieft sich wieder in sein Sachbuch über die Frage, wie die Künstliche Intelligenz den Planeten und die Medizin verändern wird. Ich denke über die letzten Minuten nach. Beginnt Künstliche Intelligenz damit, dass eine Neunzigjährige einem Halbautomaten mehr Vertrauen schenkt als ausgebildeten Ärzten? Werden Ärzte demnächst durch kommunizierende Maschinen ersetzt? Müssen wir nicht schon heute kritisch überlegen, wo wir als Berufsgruppe mit automatisierten Techniken hinwollen, und müssen wir nicht zeigen, dass alle Ärzte zuerst selbst Menschen sind, die alles für andere Menschen bereit sind zu geben? Die OP-Roboter haben Operationen nicht automatisch erfolgreicher gemacht, Komplikationen und Mortalität in Studien im Vergleich zu analog arbeitenden Chirurgen teilweise erhöht. Ich gebe zu, die neuen digitalen und automatischen Techniken sind faszinierend und können, wie in diesem Fall, Leben retten. Vor 50 Jahren wäre die junge Frau gestorben, egal ob ein Arzt da gewesen wäre, weil der Strom zum Schock aus dem Gerät gefehlt hätte.

Aus diesem Grund müssen wir Ärzte gerade in Zeiten von Automatisierung und Digitalisierung Beziehungen anbieten, wenn wir verhindern wollen, dass Patienten unserer Freundlichkeit und Kompetenz weniger vertrauen als einer Maschine. Aber vielleicht ist das alles ein frommer Wunsch? Vielleicht reicht schon eine freundliche Stimme, Zeit für Gespräche, Beziehungen zwischen Arzt und Patienten. Wenn wir uns selbst wie Roboter verhalten, besteht die Gefahr, dass uns Roboter oder Computer in Zukunft wirklich ablösen können. Das betrifft nicht nur Radiologen. Der Werbespruch der Industrie müsste demnach lauten: Nehmt die Künstliche Intelligenz an, aber vergesst nicht das Wichtigste - die Menschen.

Ich beobachte den jungen Mann gegenüber. Ich würde am liebsten mit ihm über meine Erlebnisse reden. Er legt das Buch weg, steckt Kopfhörer in die Ohren und zückt nun ebenfalls sein Natel, wischt und tippt und sendet Message um Message, an einen Menschen oder eine Maschine. Ich, sein analoges Gegenüber, hätte ihm zeigen können, zu welcher Spezies ich gehöre: Ich bin ein Mensch, ein humanes Wesen, mit meist freundlicher Stimme und vielen Zweifeln. 